Proceedings of the 2016 Winter Simulation Conference

T. M. K. Roeder, P. I. Frazier, R. Szechtman, E. Zhou, T. Huschka, and S. E. Chick, eds.

\title{
EVALUATION OF THE GENERAL APPLICABILITY OF DRAGOON FOR THE $k$-CENTER PROBLEM
}

\author{
Tobias Uhlig \\ Peter Hillmann \\ Oliver Rose \\ Department of Computer Science \\ Universität der Bundeswehr München \\ Werner-Heisenberg-Weg 39 \\ Neubiberg, 85577, GERMANY
}

\begin{abstract}
The $k$-center problem is a fundamental problem we often face when considering complex service systems. Typical challenges include the placement of warehouses in logistics or positioning of servers for content delivery networks. We previously have proposed Dragoon as an effective algorithm to approach the $k$-center problem. This paper evaluates Dragoon with a focus on potential worst case behavior in comparison to other techniques. We use an evolutionary algorithm to generate instances of the $k$-center problem that are especially challenging for Dragoon. Ultimately, our experiments confirm the previous good results of Dragoon, however, we also can reliably find scenarios where it is clearly outperformed by other approaches.
\end{abstract}

\section{INTRODUCTION}

Imagine a manager that plans new warehouses to distribute goods to his customers. Consider a planner of content delivery networks who has to setup servers close to users to provide reliable access to information. Think of an emergency staff that needs to know where to place distribution centers to supply basic goods after a natural disaster. Complex service system like these and many other scenarios are instances of the $k$-center problem. Consequently, there is a big interest in solving these problems. Especially considering the last two examples we need reliable results in short time. Accordingly, an appropriate optimization approach needs to be robust and efficient. Figure 1 shows an abstract instance of the $k$-center problem and a solution generated by the Dragoon algorithm. A general introduction to the $k$-center problem and respective optimization heuristics can be found in Kleinberg and Tardos (2005).

In this paper we discuss Dragoon in detail, an optimization approach that has previously been identified as an efficient optimization technique (Hillmann et al. 2015). We focus more on the robustness aspect

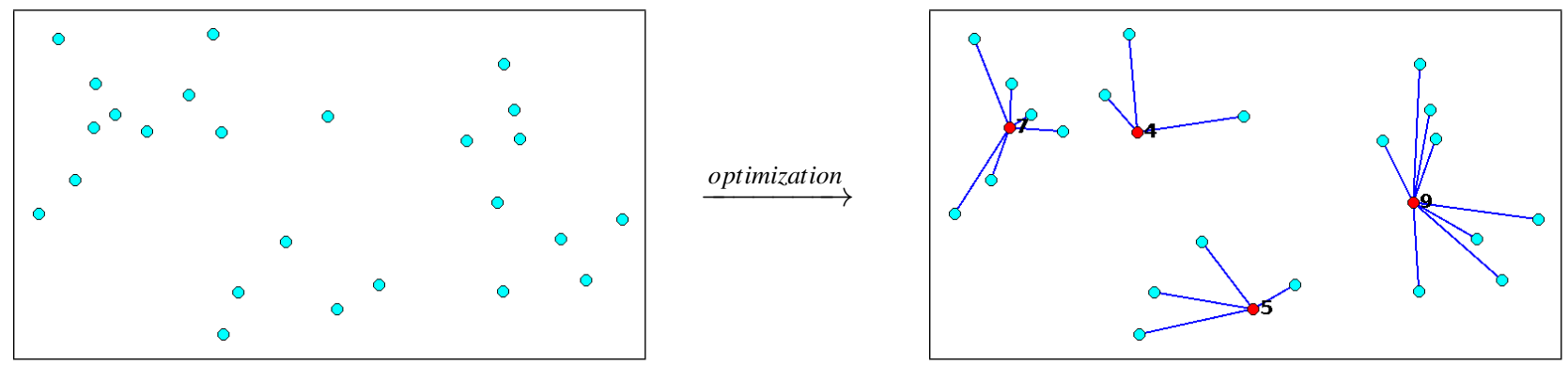

Figure 1: The $k$-center problem: for a set of customers the challenge is to determine a given number of central locations to provide a service to the customers. 
of the optimization, by analyzing its worst case behavior in comparison to other techniques. To this end we employ an evolutionary algorithm to generate problem instances that are especially challenging for Dragoon. This idea is inspired by a paper from Nguyen et al. 2014. They used an evolutionary algorithm to generate images that would fool a deep neural network. The considered deep neural network was trained to recognized certain classes of images, however, the research group was able to generate images that were false positives. Similarly, we try to identify instances of the $k$-center problem were the explicit and implicit optimization assumptions of Dragoon are exploited, leading to low quality solutions.

Our paper is structured in the following way: In the next section we briefly introduce the $k$-center problem and its characteristics. Afterward, we present the optimization approaches we considered. In Sections 4 and 5 we explain our experimental setup and provide the results of our tests. Finally, we discuss the results of our study and give a summary and short outlook.

\section{THE K-CENTER PROBLEM}

The $k$-center problem is a classic optimization problem, that is known to be NP-hard (Gonzalez 1985). Informally, we are looking to place multiple centers to satisfy a number of customers. Our goal is to minimize the maximal distance of a customer to its closest center. For example, in disaster management we want to find good locations for camps to provide services to multiple cities. More formally, given a set of locations $V$ and number of centers to place $(k)$ and a distance function $d$ we have to determine a subset $S \subseteq V$ with $|S|=k$. The optimization objective $D$ is the minimization of the maximal distance of locations to their closest center (see Equation 1). For this paper the distance function $d$ is based on the Euclidean distance in $R^{2}$.

$$
D=\min \max _{v \in V} \min _{s \in S} d(v, s)
$$

In this paper we consider only the placement of centers at a customer location (Node-Placement). We do not discuss the more general Free-Placement, where customers can be placed anywhere. The results and approaches presented in this paper can, however, be extended to consider Free-Placement. We focus on Node-Placement since it is oftentimes reasonable to rely on the existing infrastructure, e.g., given network infrastructure for CDNs or existing roads for logistics problems. Especially, for problems with a short planning horizon like disaster management or dynamic adaption of CDNs it is impractical to setup a new center, that is not at an customer location. When we consider time critical applications we need an optimization approach that is fast and robust. The following section will briefly introduce a number of optimization techniques for the $k$-center problem.

\section{OPTIMIZATION APPROACHES}

Previously, we mentioned the NP-hard nature of the $k$-center problem, which makes it a difficult problem to solve. There is no fast exact approach to calculate a solution for a given problem instance. Small instances can be solved optimally using brute force or branch and bound techniques. Larger scenarios cannot be solved practically since the search space grows exponentially. To handle these instances a number of heuristic approaches have been proposed to generate approximate solutions. Generally, as long as they have polynomial runtime these heuristics can only be guaranteed to be 2-approximable, i.e., at worst the maximal distance can be twice as large as the theoretical optimum (Kleinberg and Tardos 2005).

Dragoon is an 2-approximbale approach that generated very promising results in previous experiments (Hillmann et al. 2015). We will compare it to the following techniques: 2-Approx, MacQueen, Greedy, and a new extension of Greedy called Backtrack. A special focus is put on worst case performance of Dragoon in comparison to the reference approaches. Dragoon and the other techniques are presented in the next few sections. All of them have two things in common, they are reasonably fast and they can be implemented to operate in a nearly deterministic way without many stochastic effects. This allows us 
to perform a huge number of experiments for different problem instances, without requiring experiment repetitions for statistical significant results.

We have compared Dragoon to other approaches before, that we do not include in this paper. Typical metaheuristics like evolutionary algorithms and particle swarm optimization are left out because of their stochastic nature. Integer linear programming is excluded from the experiments since it requires to much calculation time to evaluate large instances effectively.

\subsection{Dragoon}

The Dragoon algorithm was initially used to optimize Landmark positions to improve the quality of IP geolocation (Hillmann et al. 2015a, Hillmann et al. 2015b). However, it can be applied to any kind of $k$-center problem. Dragoon uses three stages for optimization: first an initial reference placement, second center placement based on the 2-Approx algorithm (see next section), and finally an iterative improvement strategy.

For the initialization, Dragoon places a single virtual center by solving the 1-center problem. This means it determines the optimal position for a single center. This center will only be used for the first optimization step and is removed afterward, consequently, it is not part of the final solution. The advantage of this approach is that oftentimes the first center serves more customers than centers placed later on. By removing the virtual center we generate solutions that are much more balanced with regard to the numbers of customers assigned to each center.

In the second stage, Dragoon iteratively places centers using the 2-Approx strategy to position all centers. The first placement decision is incorporating the virtual center from the initialization step. Afterward, the virtual center is removed and no longer specifically considered in further placement decision. When the second stage concludes we have a guaranteed 2-approximable solution, which is then improved in the final stage.

During the final stage, a local optimization strategy is used to obtain a better solution. The procedure iterates over all centers and tries to move them to an adjacent node. If such a move leads to an improvement it is accepted, otherwise it will be undone. Consequently, the algorithm can only improve the initial solution and therefore leads to overall better result. This process is repeated until no further improvement can be obtained. In the next section we will discuss the 2-Approx approach that is used in Dragoon's second stage.

\subsection{2-Approx}

The 2-Approx algorithm discussed in Hochbaum and Shmoys (1985) and is named this way, since it reliably provides a 2-approximable solution for the maximum distance $(D)$ objective. This means, the following is true for all solutions generated by 2-Approx:

$$
D_{2-\text { Approx }} \leq D_{\text {optimal }} \cdot 2 \text {. }
$$

The algorithm uses an iterative approach for center placement. The first center is placed arbitrarily, either randomly or using some kind of initialization. Each successive center is placed at the customer location that has the maximal distance to its closest center. This procedure is repeated until all centers are positioned.

\subsection{MacQueen}

MacQueen is an implementation of a k-means approach (MacQueen 1967). Starting from an initial placement it iteratively optimizes a solution until no further improvements are achieved. For each iteration customers are assigned to their closest center. Then each center location is changed to optimally support the assigned customers. The repositioning is based on the geometrical mean of the customer group. Eventually, center positions no longer change and the optimization ends. Selim and Ismail (1984) have shown that k-means approaches are guaranteed to converge. 


\subsection{Greedy}

We use a straight forward implementation of a greedy approach discussed in Jamin et al. (2001). We iteratively place centers one at a time. To place a center, all possible locations are evaluated and the center is placed at the position that maximizes the optimization objective. For node-based placement this means, we consider all customer locations during each iteration. With regard to free placement, some kind of rasterization is required. Similar to the 2-Approx approach, Greedy also guarantees a 2-approximable result.

\subsection{Backtrack}

Backtrack is an extension of the Greedy algorithm we propose. Generally, each placement decision in Greedy is only locally optimal. Therefore, the generated solution is usually not a global optimum. Backtrack takes the solution generated by Greedy and tries to improve it. It only performs changes that lead to an improvement, consequently, it only generates solutions that are better or at least equally good as the ones generated by Greedy. For this reason, Backtrack also guarantees 2-approximable results. For the optimization Backtrack individually evaluates all centers and tries to reposition them. This is done iteratively for every available center. The optimization terminates either when we cannot find a better position for single center after we iterated over all of them or when a given number of optimization steps is reached. We have to limit the maximal number of optimization steps, since we cannot predict how long the optimization would run otherwise. In contrast to MacQueen, there is no guarantee of convergence.

\section{EXPERIMENT SETUP}

In our experiments we always consider the relative performance of two approaches to solve a given problem instance. Usually, we look at the performance of an "challenger" algorithm in comparison to a "challenged" approach (usually Dragoon). To measure the difference between them we simply calculate the absolute difference for a given problem instance:

$$
\Delta D=D_{\text {challenger }}-D_{\text {challenged }}
$$

Therefore, a negative $\Delta D$ indicates that the challenger performed better than the challenged (Dragoon).

The first part of our experiments was designed to evaluate the average performance of the algorithms. We considered six general setups of the $k$-center problem (see Table 1). Instance of these setups were generated randomly by placing the customers at random position in a square with $0.0<x, y<100.0$. We usually selected square numbers for the amount of customers and centers, since we can easily map them into the square area for thought experiments. For example, if we consider 4 centers that are placed exactly in the middle of the 4 sub-quadrants (Free Placement) of our area with side length 100.0 we know that $D$ can at most be $35.36\left(\frac{1}{4} \sqrt{100^{2}+100^{2}}\right)$. For our experiments we evaluated 1000 random instances for each comparison.

Table 1: Experiment setups

\begin{tabular}{|l|cccccc|}
\hline Setup & I & II & III & IV & V & VI \\
\hline Customers & 10 & 25 & 36 & 49 & 49 & 64 \\
Centers & 2 & 4 & 4 & 9 & 4 & 16 \\
\hline
\end{tabular}

In the second part of our experiments we tried to identify problem instance were the challenger approach would significantly outperform Dragoon. To find these instance we employed an evolutionary algorithm that we implemented using the Serein framework (Uhlig 2015). The evolutionary algorithm used the following setup: 
- Problem encoding: For $\mathrm{n}$ customers we used a vector of doubles $(0.0<z<1.0)$ containing an $\mathrm{x}$ and y component for each customer location $\vec{v}=\left(z_{1}^{x}, z_{1}^{y}, \ldots, z_{n}^{x}, z_{n}^{y}\right)$. A pair of components $z$ is mapped to a location in our square $\left(100.0^{2}\right)$.

- Fitness function: Using the mapped customer locations the centers were placed using the challenging and challenged algorithm. Afterward, the fitness was calculated as $\Delta D$

- Reproduction operators: we used single point Gaussian mutation with a standard deviation of 0.05 and whole arithmetic recombination with a probability of 0.3 .

- Selection operators: we employed tournament selection with tournament size 2 and deterministic selection for survival with uncapped elitism.

- We used a population size of 20 and run the evolutionary algorithm for 100 generations.

Starting from a random population of scenarios the evolutionary algorithm searches for a fitting scenario. In our context fitting means, a scenario that can be solved by the challenger approach with a better result than the solution provided by Dragoon. The next section will discuss the result of both experiments.

\section{RESULTS}

The first part of our experiments aimed to confirm the results of our previous studies. We expected Dragoon to perform better than the previously considered reference approaches, i.e., MacQueen, 2-Approx, and Greedy. At the same time we included the new approach Backtrack to evaluate its performance. Looking at the results in Table 2, we can confirm that Dragoon on average returns comparably good results. However, Backtrack slightly outperform Dragoon.

Table 2: Average performance of approaches in comparison to Dragoon $\left(\Delta D_{\text {avg }}\right)$ based on 1000 random problem instances (smaller values are better).

\begin{tabular}{|c|cccc|}
\hline Users / Centers & MacQueen & 2-Approx & Greedy & Backtrack \\
\hline 10 / 2 & 4.95 & 13.31 & 4.59 & -0.71 \\
$25 / 4$ & 7.64 & 9.09 & 9.5 & 0.70 \\
36 / 4 & 7.84 & 10.13 & 11.02 & -0.59 \\
49 / 9 & 6.92 & 3.72 & 6.42 & -1.23 \\
64 / 4 & 7.43 & 12.12 & 12.61 & -0.71 \\
64 / 16 & 6.10 & 1.68 & 2.77 & -1.35 \\
\hline
\end{tabular}

The second part of our experiments considered the worst case performance of Dragoon. Table 3 illustrates that we were able to determine problem instance were Dragoon failed in comparison to the reference approach. Conversely, we can say that for each approach there exist scenarios where they can significantly outperform Dragoon. For example, Figure 2 shows a scenario where the MacQueen algorithm determines a much better solution than the Dragoon approach. Interestingly Backtrack, our new approach, showed a slightly better average performance than Dragoon.

Table 3: Worst case performance of Dragoon in comparison to other approaches for a problem instance generated by a GA (smaller $\Delta D$ values are better).

\begin{tabular}{|c|cccc|}
\hline Users / Centers & MacQueen & 2-Approx & Greedy & Backtrack \\
\hline $10 / 2$ & -26.41 & -29.43 & -31.78 & -23.55 \\
$25 / 4$ & -17.34 & -10.54 & -10.81 & -13.39 \\
$36 / 4$ & -3.23 & -10.84 & -11.96 & -14.85 \\
49 / 9 & -8.75 & -5.92 & -4.92 & -8.20 \\
64 / 4 & -7.81 & -4.73 & -0.82 & -10.32 \\
$64 / 16$ & -5.49 & -5.41 & -4.65 & -6.05 \\
\hline
\end{tabular}




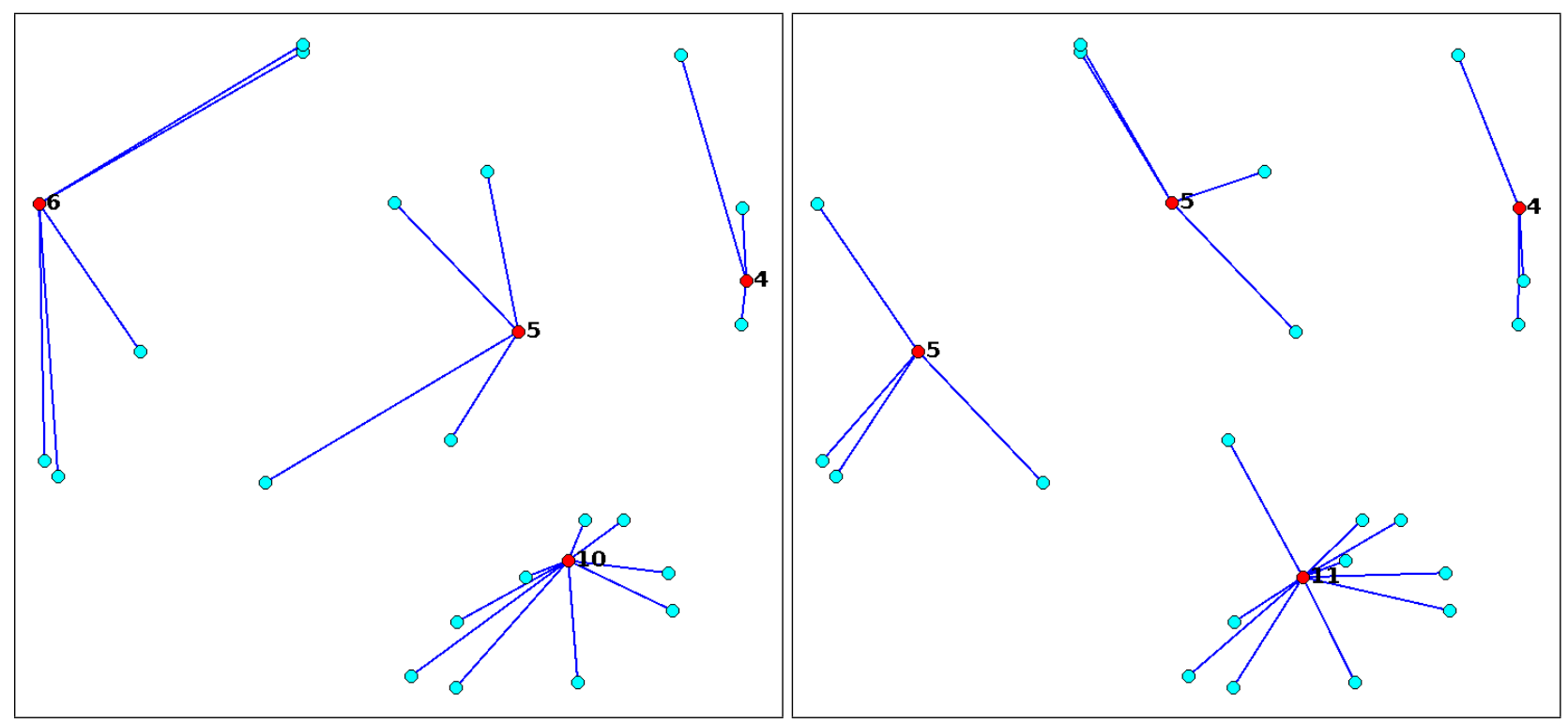

Figure 2: For the given scenario Dragoon (left) finds only a suboptimal solution. MacQueen (right) determines a much better solution.

\section{DISCUSSION}

Our results illustrate that there are worst case scenarios where Dragoon is clearly outperformed by other approaches. In critical application areas this can lead to lower quality solutions than initially expected. For these scenarios it is important to acknowledge the potential of failure and include appropriate fall-back methods. Looking at the scenarios where Dragoon performed worst, we noticed that they often had single customers which where located in isolated positions. However, currently we have no empirical data to statistically support this observation.

It is important to note, that occurrences of low quality solutions are not limited to Dragoon. Each of the presented approaches can fail given a certain problem instance. In general, nearly every approach can be beaten by all other techniques in certain constellations. Table 4 illustrates this fact by matching each approach against all the other ones. Here we show only one setup, however, the general result was the same across all configurations.

One exception is the comparison between Greedy and Backtrack, usually Greedy should not be able to outperform Backtrack. Backtrack strictly improves a solution generated by Greedy, therefore, it should be strictly superior. Looking at the results in Table 4, we see an observation that seems to contradict this guarantee, i.e, Greedy outperforms Backtrack with $\Delta D=-0.39$ ). At this point it is important to remember that we selected algorithms that are mostly deterministic, nevertheless, there is still some randomness

Table 4: Summary of results: Can a certain "challenger" approach outperform another "challenged" approach in an instance of the $k$-center problem with 49 customers and 4 centers (smaller $\Delta D$ values are better).

\begin{tabular}{|c|ccccc|}
\cline { 2 - 6 } \multicolumn{1}{c|}{} & \multicolumn{5}{c|}{ Challenged } \\
\hline Challenger & MacQueen & Backtrack & TwoApprox & Greedy & Dragoon \\
\hline MacQueen & & -6.29 & -24.38 & -22.68 & -7.81 \\
Backtrack & -32.68 & & -24.73 & -26.45 & -10.32 \\
TwoApprox & -31.26 & 0.0 & & -21.82 & -4.37 \\
Greedy & -21.54 & -0.39 & -15.47 & & -0.82 \\
Dragoon & -38.53 & -8.52 & -24.64 & -22.12 & \\
\hline
\end{tabular}


included. Specifically, for Greedy if two potential placements lead to the same improvement the one that is actually selected is chosen randomly.

In Table 4, 2-Approx was not able to outperform Backtrack, but in principle it is able to do so. For other setups we have found instances where it is better than Backtrack. Nonetheless, the fact that it did not outperform Backtrack in the given example indicates that it is quite difficult to find a instance were it is superior.

\section{SUMMARY AND OUTLOOK}

We presented a critical view at the Dragoon optimization approach, considering its potential worst case behavior. While it returns very good results on average, there are instances of the $k$-center problem where it is outperformed significantly. The novel Backtrack approach generated very good results and is even slightly better than Dragoon. However, as discussed before, it is also susceptible to fail for certain scenarios. The general verdict is that all approaches shine for certain scenarios and fall off in other cases.

This paper considered the $k$-center problem on a very abstract level. Our future work will focus more on practical aspects of the problem. For example, like nonlinear distance calculation between customer and its center including factors like dynamic traffic, limited availability of certain services depending on the center. This includes more intricate assignment strategies that match customers and centers based on customer profiles.

With regard to the underlying optimization there are three potential paths to follow. The first and simplest approach to guarantee more robustness is to use more than one of the presented optimization techniques, to limit the occurrence of worst case behavior. Considering time critical application this path might, however, be impossible. As a second path, we can add some intelligence to select the appropriate approach for a given problem instance. We could either try to train a neural network to classify the problem instances or strive to identify certain problem properties that favor certain approaches, e.g., avoiding Dragoon for problems were certain customers have secluded positions. Finally, we are also investigating opportunities to further increase the robustness of the approaches on an algorithmic level. Currently we are developing an advanced version of Dragoon.

\section{REFERENCES}

Gonzalez, T. F. 1985. "Clustering to Minimize the Maximum Intercluster Distance". Theoretical Computer Science 38:293 - 306.

Hillmann, P., L. Stiemert, G. D. Rodosek, and O. Rose. 2015a, Dec. "Dragoon: Advanced Modelling of IP Geolocation by Use of Latency Measurements". In 2015 10th International Conference for Internet Technology and Secured Transactions (ICITST), 438-445.

Hillmann, P., L. Stiemert, G. D. Rodosek, and O. Rose. 2015b, Nov. "Modelling of IP Geolocation by Use of Latency Measurements". In Network and Service Management (CNSM), 2015 11th International Conference on, 173-177.

Hillmann, P., T. Uhlig, G. D. Rodosek, and O. Rose. 2015, Nov. "A Novel Approach to Solve k-Center Problems with Geographical Placement". In Service Operations and Logistics, and Informatics (SOLI), 2015 IEEE International Conference on, 31-36.

Hochbaum, D. S., and D. B. Shmoys. 1985. "A Best Possible Heuristic for the $k$-Center Problem". Mathematics of Operations Research 10:180-184.

Jamin, S., C. Jin, A. R. Kurc, D. Raz, and Y. Shavitt. 2001. "Constrained Mirror Placement on the Internet". In INFOCOM 2001. Twentieth Annual Joint Conference of the IEEE Computer and Communications Societies, Volume 1, 31-40.

Kleinberg, J., and E. Tardos. 2005. Algorithm Design. Boston, MA, USA: Addison-Wesley Longman Publishing Co., Inc. 
MacQueen, J. 1967. "Some Methods for Classification and Analysis of Multivariate Observations". In Proceedings of the Fifth Berkeley Symposium on Mathematical Statistics and Probability, Volume 1: Statistics, 281-297.

Nguyen, A. M., J. Yosinski, and J. Clune. 2014. "Deep Neural Networks Are Easily Fooled: High Confidence Predictions for Unrecognizable Images". Computer Vision and Pattern Recognition abs/1412.1897.

Selim, S. Z., and M. A. Ismail. 1984. " $k$-Means-Type Algorithms: A Generalized Convergence Theorem and Characterization of Local Optimality". IEEE Transactions on Pattern Analysis and Machine Intelligence 6 (1): 81-87.

Uhlig, T. 2015. Self-Replicating Individuals. München: Dr. Hut.

\section{AUTHOR BIOGRAPHIES}

TOBIAS UHLIG is a researcher at the Universität der Bundeswehr München, Germany. He received his M.S. degree in computer science from Dresden University of Technology and a Ph.D. degree in computer science from the Universität der Bundeswehr München. His research interests include metaheuristics, simulation based optimization, and process modeling. He is a member of the ASIM and IEEE RAS Technical Committee on Semiconductor Manufacturing Automation. His email address is tobias.uhlig@unibw.de.

PETER HILLMANN is a Ph.D. student at the Universität der Bundeswehr München, Germany. He holds a M.Sc. in Information-System-Technology from Dresden University of Technology since 2011. His areas of research are network and system security with focus on cryptography as well as scheduling and optimization problems. His email address is peter.hillmann@unibw.de.

OLIVER ROSE holds the Chair for Modeling and Simulation at the Department of Computer Science of the Universität der Bundeswehr München, Germany. He received an M.S. degree in applied mathematics and a Ph.D. degree in computer science from Würzburg University, Germany. His research focuses on the operational modeling, analysis and material flow control of complex manufacturing facilities, in particular, semiconductor factories. He is a member of IEEE, INFORMS Simulation Society, ASIM, and GI. His email address is oliver.rose@unibw.de. 